\title{
Complementary Iterated Floor Words and the Flora Game
}

\author{
Aviezri S. Fraenkel* \\ Department of Computer Science and Applied Mathematics \\ Weizmann Institute of Science \\ Rehovot 76100, Israel
}

September 6, 2009

\begin{abstract}
Let $\varphi=(1+\sqrt{5}) / 2$ denote the golden section. We investigate relationships between unbounded iterations of the floor function applied to various combinations of $\varphi$ and $\varphi^{2}$. We use them to formulate an algebraic polynomial-time winning strategy for a new 4-pile take-away game Flora, which is motivated by partitioning the set of games into subsets CompGames and PrimGames. We present recursive, arithmetic and word-mapping winning strategies for it. The arithmetic one is based on the Fibonacci numeration system. We further show how to generate the floor words induced by the iterations using word-mappings and and characterize them using the Fibonacci numeration system. We also exhibit an infinite array of such sequences.

Keywords: floor function, integer part function, combinatorics of words, combinatorial game theory, Fibonacci numeration system

Mathematics Subject Classification: 11B75, 11B39, 91A46
\end{abstract}

\section{Introduction}

As customary, we denote by $\lfloor x\rfloor$ the integer part of $x$, commonly known as the floor function. It is the largest integer not exceeding $x$. Let $\varphi=(1+\sqrt{5}) / 2$ denote the golden section.

Two topics motivate this work. On the one hand, we wish to study what happens when we keep iterating the floor function with either $\varphi$ or $\varphi^{2}$ in various ways. Are any interesting relationships between them discernible even after an unbounded number of iterations, or total chaos takes over?

On the other hand, we aim at shedding more light on the class of impartial take-away games. This class appears to be partitioned into two disjoint

*fraenkel@wisdom.weizmann.ac.il http://www.wisdom.weizmann.ac.il/ fraenkel 
subclasses: those that are easy to generalize to more than one or two piles, and those for which this seems to be very hard. A well-known representative of the former is Nim [2], and of the latter Wythoff's game [5]. Some progress in generalizing Wythoff to multiple piles was recently made. See [9], [24], [23]. Three-pile games that are extensions rather than generalizations of Wythoff were also given recently [8], [4].

Here we consider an extension of Wythoff to four piles. The efforts in defining a "right" extension and particularly in proving the validity of the winning strategy are considerably greater than those for three-pile extensions. We present four formulations of the winning strategy.

In $\S 2$ we investigate unbounded iterations of the floor function and formulate a wealth of relationships and identities. In $\S 3$ we define the subclasses CompGames and PrimGames, which motivate the definition of the 4-pile game dubbed Flora. In $\S 3.1$ we formulate an algebraic winning strategy for the game, based on the results derived in $\S 2$, and prove that its complexity is poly-time. In $§ 3.2$ we formulate a recursive winning strategy which appears very simple, but its polynomiality is implied only by a recent result [12]. We end in $\S 3.3$ with a polynomial-time arithmetic winning strategy, based on the Fibonacci numeration system. In $\S 4$ we indicate how to generate sequences induced by iterations of the floor function using word-mappings. We apply it to one of the sequences in $\S 4.1$. In $\S 4.2$ we present our fourth, word-mapping, formulation of the winning strategy of the Flora game, which is also polynomial-time. In $\S 4.3$ we use results from $\S 3.3$ and $\S 2$ and make minor use of the language of $\S 4$, to characterize the representations of general cases of the special sequences playing a major role in the algebraic formulation of the winning strategy of Flora. In $\S 5$ we show, by means of an example, how to produce infinite complementary arrays using sequences induced by the iterations. In the final Epilogue we wrap up and indicate natural further directions of research.

Let $a(n)=\lfloor n \varphi\rfloor, b(n)=\left\lfloor n \varphi^{2}\right\rfloor$. It is well-known that the sequences $a$ and $b$ split the positive integers [5], $\S 3$. An example of an iterated identity is $a(b(n))=a(n)+b(n)$. It can be abbreviated as $a b=a+b$, where the product means iteration (composition). We also write $a^{2}$ for $a a, a b^{3} a^{2}$ for abbbaa, etc. An example of 4 iterated complementary sequences is $a^{2}=b-1, a b=a+b$, $b a=a+b-1, b^{2}=a+2 b$, since every positive integer is in precisely one of these 4 sequences. We use the notation $w=w_{1} w_{2} \ldots w_{k}$ to denote the word $w$ as well as the (iterated) sequence $w(n)$. If the sequence is intended, we sometimes write $w(n)$ rather than only $w$. Notice that the product, though not commutative, is associative.

Let $h=b, u=a$, and for $k \geq 2, h^{k}=a^{k-1} b, u^{k}=b a^{k-1}$. Let $\Delta a^{k}(n)=$ $a^{k}(n+1)-a^{k}(n), \Delta h^{k}(n)=h^{k}(n+1)-h^{k}(n)$. For technical reasons we put

$$
a^{0}(n)=n, \quad h^{0}(n)=a(n) .
$$

Further, let $F_{-1}=1, F_{0}=1, F_{n}=F_{n-1}+F_{n-2}(n \geq 1)$ be the Fibonacci sequence. 
Notation 1. For $k \geq 0, s \in \mathbb{Z}$, let $G_{k}=\cup_{n=1}^{\infty} a^{k}(n), H_{k}=\cup_{n=1}^{\infty} h^{k}(n), U_{k}=$ $\cup_{n=1}^{\infty} u^{k}(n), V_{2}=\cup_{n=1}^{\infty} b^{2}(n), G_{k}-s=\cup_{n=1}^{\infty}\left(a^{k}(n)-s\right.$ ) (subtracting $s$ from every element of $\left.G_{k}\right)$.

In particular, $G_{0}=\mathbb{Z}_{\geq 1}$, and $H_{0}=G_{1}=U_{1}$.

Note. In our applications, $s \in\{0,-1,-2,2\}$, most often 0 .

\section{Identities}

After multiplying by the irrational $\varphi$ and then throwing out the fractional part for an unbounded number of times, one might expect complete chaos among relationships involving $a^{k}, h^{k}, u^{k}$ and $b^{k}$. It is thus surprising that there are many striking identities and relationships among them. Our purpose in this section is to prove a selection of them.

Theorem 1. For every $k \in \mathbb{Z}_{\geq 1}$ and every $n \in \mathbb{Z}_{\geq 1}$ the following holds:

(a) The $k+1$ sequences $G_{k}, H_{k}, H_{k-1}, \ldots, H_{2}, H_{1}$ partition $\mathbb{Z}_{\geq 1}$.

(b) $u^{k+1}=a^{k}+a^{k+1}=a^{k+2}+1$.

(c) $h^{k}=a^{k+1}+F_{k-1}$.

(d) $u^{k+1}=h^{k+1}-F_{k}+1=a^{k+2}+1$.

(e) (e1) $h^{k+1}-h^{k}=a^{k}+F_{k-2}-1$.

(e2) $h^{k+1}-a^{k+1}=a^{k}+F_{k}-1$.

(e3) $a u^{k+1}=u^{k+2}+1$.

(f) (f1) Let

$$
S_{1}=\left\{n \in \mathbb{Z}_{\geq 1}: \Delta a(n)=F_{0}\right\}, \quad S_{2}=\left\{n \in \mathbb{Z}_{\geq 1}: \Delta a(n)=F_{1}\right\} .
$$

Then $S_{1}$ and $S_{2}$ split $\mathbb{Z}_{\geq 1}$; and each of $S_{1}$ and $S_{2}$ is an infinite set.

(f2) For all $k \in \mathbb{Z}_{\geq 1}$ the following holds: $\Delta a^{k}(n)=F_{k-1}$ for all $n \in S_{1}$ and $\Delta a^{k}(n)=F_{k}$ for all $n \in S_{2}$.

(f3) (i) If $\Delta a^{k}(n+1)=F_{k-1}$ for some $n \in \mathbb{Z}_{\geq 1}$, then $\Delta a^{k}(n)=\Delta a^{k}(n+$ 2) $=F_{k}$. (ii) If $\Delta a^{k}(n+1)=\Delta a^{k}(n+2)=F_{k}$ for some $n \in \mathbb{Z}_{\geq 1}$, then $\Delta a^{k}(n)=\Delta a^{k}(n+3)=F_{k-1}$.

(f4) $\Delta a^{k}(n)=\Delta h^{k-1}(n) \in\left\{F_{k-1}, F_{k}\right\}$, and each of $F_{k-1}$ and $F_{k}$ is assumed for infinitely many $n$.

(f5) (i) $\Delta a^{k}(0)=1, \quad$ (ii) $\Delta h^{k}(0)=F_{k-1}+1$.

(g) $a^{k}(h(n))=h^{k+1}(n)$ (due to Lior Goldberg).

(h) (h1) $\left(G_{2}+2\right) \subset G_{1}$. 
(h2) $G_{2} \cup\left(G_{2}+2\right)=G_{1}$.

(h3) $U_{2} \subset\left(G_{1}-2\right) \subset G_{1} \cup U_{2}$.

(h4) $\left(V_{2}-1\right) \subset G_{2}$.

We begin by recalling some elementary properties of the floor function. Let $x$, $y$ be any real numbers. Denote by $\{x\}$ the fractional part of $x$, so $x=\lfloor x\rfloor+\{x\}$. Then:

- $0 \leq\{x\}<1, x-1<\lfloor x\rfloor \leq x$. Replacing $x$ by $-x,-x-1<\lfloor-x\rfloor \leq-x$, hence $-1 \leq\lfloor x\rfloor+\lfloor-x\rfloor \leq 0$ and $\lfloor x\rfloor+\lfloor-x\rfloor=0$ if and only if $x$ is an integer. For example, $\lfloor\varphi\rfloor=1,\lfloor-\varphi\rfloor=-2,\lfloor\varphi\rfloor+\lfloor-\varphi\rfloor=-1$; and $\varphi^{2}=\varphi+1$ implies $\{\varphi\}=\varphi^{-1}=\varphi-1$.

- $\lfloor x\rfloor+\lfloor y\rfloor \leq\lfloor x+y\rfloor \leq\lfloor x\rfloor+\lfloor y\rfloor+1$. This follows immediately from $\lfloor x+y\rfloor=\lfloor\lfloor x\rfloor+\{x\}+\lfloor y\rfloor+\{y\}\rfloor=\lfloor x\rfloor+\lfloor y\rfloor+\lfloor\{x\}+\{y\}\rfloor$.

Lemma 1. (i) Let $s \in \mathbb{Z}$. Each of the sequences $G_{k}+s, H_{k}+s, U_{k}+s, V_{2}+s$ is strictly increasing for every $k \geq 1$.

(ii) The sequences $G_{k}, H_{k}$ split $G_{k-1}$ for every $k \geq 1$.

Proof. (i) Follows from the fact that $\varphi^{2}=\varphi+1>\varphi>1$.

(ii) Since $\varphi^{-1}+\varphi^{-2}=1$, the sequences $G_{1}$ and $H_{1}$ split $\mathbb{Z}_{\geq 1}=G_{0}$ (see e.g., [5], §3), so the result holds for $k=1$. For any $k \geq 1$, assume that $G_{k}, H_{k}$ split $G_{k-1}$. Then

$$
\begin{aligned}
G_{k+1} \cup H_{k+1} & =\bigcup_{n}\left(a^{k+1}(n) \cup h^{k+1}(n)\right)=\bigcup_{n}\left(a a^{k}(n) \cup a h^{k}(n)\right) \\
& =\bigcup_{n} a a^{k-1}(n) \text { (by induction) }=\bigcup_{n} a^{k}(n)=G_{k} .
\end{aligned}
$$

Proof of Theorem 1 (a). We noted that $G_{1}$ and $H_{1}$ split $\mathbb{Z}_{\geq 1}$. Suppose that $G_{k}, H_{k}, H_{k-1}, \ldots, H_{2}, H_{1}$ partition $\mathbb{Z}_{\geq 1}$. Then $G_{k+1}, H_{k+1}, H_{k}, \ldots, H_{2}, H_{1}$ partition $\mathbb{Z}_{\geq 1}$, since $G_{k+1}, H_{k+1}$ split $G_{k}$ by Lemma 1 .

Note. It follows from Lemma 1(ii) (or from (a)) that for any positive integers $m, n, a(m) \neq b(n)$. This property will be referred to in the sequel as disjointness.

Proof of Theorem 1 (b). By definition,

$$
a^{k+2}=a^{2} a^{k}=\left\lfloor\varphi\left\lfloor\varphi a^{k}\right\rfloor\right\rfloor \leq\left\lfloor\varphi^{2} a^{k}\right\rfloor=b a^{k}=u^{k+1} .
$$

By disjointness, $u^{k+1}=b a^{k} \geq a^{k+2}+1$. Conversely, multiply $\varphi a^{k}<a^{k+1}+1$ by $\varphi$ to get, $\varphi^{2} a^{k}<\varphi\left(a^{k+1}+1\right)$, hence $b a^{k} \leq\left\lfloor\varphi\left(a^{k+1}+1\right)\right\rfloor$. By disjointness this inequality is strict, so

$$
u^{k+1} \leq\left\lfloor\varphi\left(a^{k+1}+1\right)\right\rfloor-1 \leq a^{k+2}+\lfloor\varphi\rfloor=a^{k+2}+1 .
$$

On the other hand, $u^{k+1}=\left\lfloor(\varphi+1) a^{k}\right\rfloor=a^{k}+a^{k+1}$. 
Lemma 2. For every $k \in \mathbb{Z}_{\geq 1}$,

(i) $\left\lfloor\varphi F_{k-1}\right\rfloor \in\left\{F_{k}-1, F_{k}\right\}$.

(ii) $\left\lfloor\varphi^{2} F_{2 k-2}\right\rfloor=F_{2 k}-1,\left\lfloor F_{2 k-1} \varphi^{2}\right\rfloor=F_{2 k+1}$.

Proof. (i) The ratios $F_{k} / F_{k-1}$ are the convergents of the simple continued fraction expansion of $\varphi=[1,1,1, \ldots]$. Therefore $\left|\varphi F_{k-1}-F_{k}\right|<F_{k-1}^{-1}$ (see e.g., [14], ch. 10), so $\varphi F_{k-1}-F_{k}=\delta$, where $-F_{k-1}^{-1}<\delta<F_{k-1}^{-1}$. Thus $\left\lfloor\varphi F_{k-1}\right\rfloor=$ $F_{k}+\lfloor\delta\rfloor$. The result follows if $|\delta|<1$, which is the case for all $k \geq 1$, since $F_{k-1} \geq F_{0}=1$.

(ii) The ratios $F_{k+2} / F_{k}$ are the convergents of the simple continued fraction expansion of $\varphi^{2}=[2,1,1,1, \ldots]$. In fact, $F_{2 k+1} / F_{2 k-1}<\varphi^{2}<F_{2 k} / F_{2 k-2}$. This follows easily from [14], ch. 10. Then $\varphi^{2} F_{2 k-1}-F_{2 k+1}=\delta$, where $0<\delta<$ $F_{2 k-1}^{-1}$, hence $\left\lfloor\varphi^{2} F_{2 k-1}\right\rfloor=F_{2 k+1}$, since $0<\delta<1$ for all $k \geq 1$. Similarly, $\varphi^{2} F_{2 k-2}-F_{2 k}=\delta$, where $-F_{2 k-2}^{-1}<\delta<0$. Thus $\left\lfloor\varphi^{2} F_{2 k-2}\right\rfloor=F_{2 k}-1$, since $-1<\delta<0$ for all $k \geq 1$.

Lemma 3. $h^{2}-a^{3}=2$.

Proof. In Lemma 9 of [8] we proved the special case $k=1$ of (d), namely $h^{2}=u^{2}+1$. Thus $h^{2}-a^{3}=u^{2}-a^{3}+1$. Clearly $a^{3}=\lfloor\varphi\lfloor\varphi\lfloor n \varphi\rfloor\rfloor\rfloor \leq\left\lfloor\varphi^{2} a\right\rfloor=u^{2}$. But this inequality is strict by disjointness. Thus $h^{2}-a^{3} \geq 2$.

Conversely, multiply the inequality $\varphi a<a^{2}+1$ by $\varphi$, to get $\varphi^{2} a<\varphi\left(a^{2}+1\right)$. Therefore $\left\lfloor\varphi^{2} a\right\rfloor \leq\left\lfloor\varphi\left(a^{2}+1\right)\right\rfloor$. Again by disjointness, this inequality is strict, i.e., $u^{2} \leq a^{3}+1$. As we saw, Lemma 9 of [8] asserts $u^{2}=h^{2}-1$. Therefore, $h^{2}-a^{3} \leq 2$.

Notation 2. For any positive integer $N$, denote by $R(N)$ the representation of $N$ in the Fibonacci numeration system. It has the form $R(N)=\left(d_{m}, \ldots, d_{0}\right)$ where $N=\sum_{i=0}^{m} d_{i} F_{i}, \quad d_{i} \in\{0,1\}, \quad d_{i}=1 \Longrightarrow d_{i-1}=0, \quad i \geq 1$ [6]. The position of a representation is the subscript $i$ of $d_{i}$. Thus, $d_{0}$ is in position $0, d_{1}$ in position 1 , etc.

Proof of Theorem 1 (c). For $k=1$, this is Lemma 5 of [8], Sect. 5. For $k=2$, it is Lemma 3 above. Suppose that $h^{k}=a^{k+1}+F_{k-1}$ for some arbitrary $k \geq 2$. Multiply by $\varphi$ and take the floor of both sides. This gives, by Lemma 2 , $h^{k+1}=\left\lfloor\varphi\left(a^{k+1}+F_{k-1}\right)\right\rfloor \leq a^{k+2}+\left\lfloor\varphi F_{k-1}\right\rfloor+1 \leq a^{k+2}+F_{k}+1$. Now [8] Sect. 6 implies that $R\left(a^{2}\right)$ ends in 01. By Lemma 1, the same holds for $a^{k}$ and $h^{k}$ for every $k \geq 3$ (but it does not hold for $h^{2}$ ). Since $R\left(F_{k}\right)$ ends in 00 for $k \geq 2, R\left(a^{k+2}+F_{k}\right)$ also ends in 01 for $k \geq 2$, and so does $h^{k+1}$ for $k \geq 2$. But $R\left(a^{k+2}+F_{k}+1\right)$ ends in 10. Hence $h^{k+1}=a^{k+2}+F_{k}$.

Proof of Theorem 1 (d). From (b) and (c), $u^{k+1}=a^{k+2}+1=h^{k+1}-$ $F_{k}+1$. The second follows once more from $(\mathbf{c})$.

We note that inspection shows that (d) does not hold for $k<1$.

Proof of Theorem 1 (e1). Subtracting (c) from (c) with $k$ replaced by $k+1$ gives $h^{k+1}-h^{k}=a^{k+2}-a^{k+1}+F_{k-2}$. Substituting the value of $a^{k+2}$ 
from (b) yields the desired result.

(e2) Follows from (e1), where we replace $h^{k}$ by its value from (c).

(e3) We have,

$$
\begin{aligned}
a u^{k+1} & \left.=\left\lfloor\varphi\left\lfloor\varphi^{2} a^{k}\right)\right\rfloor\right\rfloor=\left\lfloor\varphi\left(a^{k}+a^{k+1}\right)\right\rfloor \\
& \leq a^{k+1}+a^{k+2}+1=u^{k+2}+1
\end{aligned}
$$

where the last equality follows from (b). On the other hand,

$$
\left\lfloor\varphi\left(a^{k}+a^{k+1}\right)\right\rfloor \geq a^{k+1}+a^{k+2}=u^{k+2} .
$$

Hence by disjointness, $\left\lfloor\varphi u^{k+1}\right\rfloor=u^{k+2}+1$.

We recall the following special case of Lemma 2 of [5]:

Lemma I. For integers $i>j \geq 0$ and integer $N_{i+1} \in \mathbb{Z} \geq 1$, let $R\left(N_{i+1}\right)=$ $F_{i}+F_{i-2}+\cdots+F_{j}$, where $j=0$ if $i$ is even, $j=1$ if $i$ is odd. Then $N_{i+1}=$ $F_{i+1}-1$.

This is the analog in the Fibonacci numeration system of the decimal $99 \ldots 9$.

Proof of Theorem 1 (f1). For any $n \in \mathbb{Z}_{\geq 1}$, clearly $\varphi-1<\Delta a(n)<$ $\varphi+1$, so $\Delta a(n) \in\{1,2\}=\left\{F_{0}, F_{1}\right\}$. This shows already that $S_{1}, S_{2}$ split $\mathbb{Z}_{\geq 1}$. Moreover, if $\Delta a(n)=1$ for all large $n$, then, since $h(n)$ is increasing, we would have $a(n) \cap h(n) \neq \emptyset$ for infinitely many $n \in \mathbb{Z}_{\geq 1}$, contradicting the complementarity of the 2 sequences. If $\Delta a(n)=2$ for all large $n$, then also $\Delta h(n)=2$ for all large $n$ by complementarity. But a direct computation shows that $\Delta a(n)=2 \Longrightarrow \Delta h(n)=3$, another contradiction. Thus each of $S_{1}$ and $S_{2}$ is infinite as claimed.

(f2) We proceed by induction on $k$. Suppose that for some $k \geq 1, \Delta a^{k}(n)=$ $F_{k-1}$ for all $n \in S_{1}$, and $\Delta a^{k}(n)=F_{k}$ for all $n \in S_{2}$. This holds for $k=1$ by (f1). For now let's assume that $n \in S_{1}$. Then

$$
\Delta a^{k+1}(n)=\left\lfloor\varphi a^{k}(n+1)\right\rfloor-\left\lfloor\varphi a^{k}(n)\right\rfloor<\varphi a^{k}(n+1)-\varphi a^{k}(n)+1=\varphi F_{k-1}+1
$$

by the induction hypothesis. Also,

$$
\Delta a^{k+1}(n)>\varphi a^{k}(n+1)-\varphi a^{k}(n)-1=\varphi F_{k-1}-1 .
$$

So $\left\lfloor\varphi F_{k-1}\right\rfloor \leq \Delta a^{k+1}(n) \leq\left\lfloor\varphi F_{k-1}\right\rfloor+1$. Then Lemma 2 implies that $\Delta a^{k}(n) \in$ $\left\{F_{k}-1, F_{k}, F_{k}+1\right\}$.

In the proof of (c) above, it was mentioned that $R\left(a^{2}(n+2)\right)$ ends in 01 . The same thus holds for $R\left(a^{k+1}(n+1)\right)$ and $R\left(a^{k+1}(n)\right)$ for all $k \geq 1$, since $G_{k+1}$ is a subsequence of $G_{2}$ for all $k \geq 1$. Therefore $R\left(\Delta a^{k+1}(n)\right)$ ends in 00 , the same as $R\left(F_{k}\right)$. But $R\left(F_{k}+1\right)$ ends in 01, and Lemma I implies that $R\left(F_{k}-1\right)$ ends in 10 , or in 01 , depending on whether $k$ is even or odd. Hence $\Delta a^{k+1}(n)=F_{k}$ for all $n \in S_{1}$. The same proof shows that $\Delta a^{k+1}(n)=F_{k+1}$ for all $n \in S_{2}$.

(f3) This follows easily for $k=1$ by considering the size of $\varphi$. For all $k \geq 1$ it follows from (f2). 
(f4). This follows directly from (f1) and (f2).

Note. Part of the proof of (f4) follows directly from (d):

$$
h^{k+1}(n+1)-a^{k+2}(n+1)=h^{k+1}(n)-a^{k+2}(n)=F_{k} .
$$

Hence $\Delta h^{k+1}(n)=\Delta a^{k+2}(n)$. But this establishes the equality part of (f4) only for $k \geq 3$ and doesn't prove the membership part.

(f5). (i) Induction on $k$. (ii) By definition, $\Delta h^{k}(0)=h^{k}(1)-h^{k}(0)=h^{k}(1)$. The result for $h^{k}(1)$ follows directly from (i) and (c).

(g). For $k=1, h^{2}(n)=\lfloor\varphi h(n)\rfloor=a(h(n))$. If the assertion holds for any $k \geq 1$, then $h^{k+2}(n)=\left\lfloor\varphi h^{k+1}(n)\right\rfloor=\left\lfloor\varphi a^{k}(h(n))\right\rfloor=a^{k+1}(h(n))$.

(h1). Clearly $G_{2} \subset G_{1}$, so for every $n \in \mathbb{Z}_{\geq 1}, a^{2}(n)=a(m)$ for some $m \in \mathbb{Z}_{>1}$. By (c), $a^{2}(n)+1=h(n) \notin G_{1}$. But then $a^{2}(n)+2=a(m+1) \in G_{1}$ by (f4) for $k=1$.

The following is a special case of Property 1, Sect. 5 of [5]:

Lemma II. The set of numbers $\left\{R(N): N \in G_{1}\right\}$ ends in an even (possibly $0)$ number of $0 s$, hence the complementary set of numbers $\left\{R(N): N \in H_{1}\right\}$ ends in an odd number of $0 s$.

(h2). By (h1), $G_{2} \cup\left(G_{2}+2\right) \subseteq G_{1}$. Choose any $a(n) \in G_{1}$. If $a(n) \in$ $\left(G_{2}+2\right)$, we are done. So suppose that $a(n)=a^{2}(m)+2$ for no $m \in \mathbb{Z}_{\geq 1}$. By (c), $a^{2}(m)+1=h(m)$ for all $m \in \mathbb{Z}_{\geq 1}$, so by disjointness, $a(n)=a^{2}(m)+1$ for no $m \in \mathbb{Z}_{\geq 1}$. But then $a(n)=g^{2}(m)$ for some $m \in \mathbb{Z}_{\geq 1}$ by (f4) for $k=1$, so $a(n) \in G_{2}$.

(h3). The following is immediately implied by (f3): (a) if $a(n)-1 \notin G_{1}$, then $a(n)-2 \in G_{1}$; and, conversely, (b) if $a(n)-1 \in G_{1}$, then $a(n)-2 \notin G_{1}$. Consider case (b). Lemma II then implies that $R(a(n)-1)$ ends in an even positive number of $0 \mathrm{~s}$, and $R(a(n))$ ends in 01. By Lemma I, $R(a(n)-2)$ then ends in 10. We now show that $R\left(\left\lfloor\varphi^{2} a(n)\right\rfloor\right)$ ends in 10 for all $n \in \mathbb{Z}_{>1}$.

Now $R(a(n))$ ends in $F_{2 k-2}$ for some $k \in \mathbb{Z}_{\geq 1}$. By Lemma $2(\mathrm{ii}),\left\lfloor\varphi^{2} F_{2 k-2}\right\rfloor=$ $F_{2 k}-1$, and $R\left(F_{2 k}-1\right)$ ends in 10 by Lemma I, the same as $R(a(n)-2)$ for case (b). This proves that $R\left(\left\lfloor\varphi^{2} a(n)\right\rfloor\right)$ ends in 10 for all $n \in \mathbb{Z}_{\geq 1}$, and the right-hand-side of (h3). On the other hand, let $N \in U_{2}$. Then $R(N)$ ends in 10 , and so $N+1$ and $N+2$ are both in $G_{1}$. Thus $N \in G_{1}-2$, proving the left-hand-side of (h3).

(h4). In the proof of (h3) we showed that $R\left(\left\lfloor\varphi^{2} a(n)\right\rfloor\right)$ ends in 10 for all $n \in \mathbb{Z}_{>1}$. Since $R(h(n))$ ends in an odd number of 1 s for all $n \in \mathbb{Z}_{>1}$ by Lemma II, $R\left(v^{2}(n)\right)$ ends in an odd number $N \geq 3$ of 1 's. Then Lemma I implies that $R\left(v^{2}(n)-1\right)$ ends in 01 . Theorem 3 of [8] states that $R\left(G_{2}\right)$ is the set of all numbers whose representation ends in 01 , so $\left(V_{2}-1\right) \subset G_{2}$.

Remark. Consider the word $w=\ell_{1} \ell_{2} \ldots \ell_{k}$ of length $k$ over the binary alphabet $\{a, b\}$. The number $m$ of occurrences of the letter $b$ is the weight of $w$. We also put $F_{-2}=0$. Recently, Clark Kimberling [18] proved the following nice and elegant result: 
Theorem I. For $k \geq 2$, let $w=\ell_{1} \ell_{2} \ldots \ell_{k}$ of length $k$ be any word over $\{a, b\}$ of length $k$ and weight $m$. Then $w=F_{k+m-4} a+F_{k+m-3} b-c$, where $c=F_{k+m-1}-w(1) \geq 0$ is independent of $n$.

Notice that in the theorem only the weight $m$ appears, not the locations within $w$ where the $b$ s appear. The locations, however, obviously influence the behavior of $w$. This influence is hidden in the "constant" $c=c_{k, m}$, where not only $F_{k+m-1}$ obviously depends on $k$ and $m$, but also $w(1)$.

We could have used Theorem I to prove most of the results of Theorem 1 simply by expressing each side of an identity as in Theorem I and verifying that they are identical. This verification, however, seems less satisfactory than the above proofs, which shed some light on the nature of the identities. In a recent book review it says, "but it is fair to say that while it is a proof, it is not an explanation" (AMM, 116, Aug-Sept 2009, p. 660). Hardy [13], writing about seven proofs of the Rogers-Ramanujan identities, put it this way: "None of these proofs can be called "simple" and "straightforward", since the simplest are essentially verifications". I got the Hardy reference from opinion 90 on the webpage of my esteemed opinionated friend Doron Zeilberger http://www.math.rutgers.edu/ zeilberg/OPINIONS.html. Moreover, the computation of $c$ is not, generally, so easy, as acknowledged by Kimberling. For example, we can show that for $w=h^{k}(m=1)$ we get $w(1)=h^{k}(1)=F_{k-1}+1$, so $c_{k}=F_{k-2}-1$. The proof depends on Lemma 2 and the Fibonacci numeration system.

\section{An Application: The Flora Game}

The class of impartial take-away games appears to be partitioned into two disjoint subclasses:

- CompGames (composite games), and

- PrimGames (prime games). ${ }^{1}$

Informally, CompGames are games that are easy to generalize to more than one or two piles; PrimGames are those for which this seems to be very hard. A well-known representative of the former is Nim, and of the latter, Wythoff's game. Some progress in generalizing Wythoff to multiple piles was recently made. Two 3-pile games that are extensions rather than generalizations of Wythoff were also given recently. It appears that, largely, a game belongs to class CompGames if it decomposes into a disjunctive sum of subgames, such as Nim, which is the Nim-sum of its pile sizes; and it belongs to class PrimGames if it is not decomposable. Hence the names CompGames (composite games) and PrimGames (not decomposable - prime). Whereas for the former there are theories for both the impartial as well as for the partizan case, there is no general theory for the latter yet, and we believe that these "lone wolf" games should be investigated more seriously.

\footnotetext{
${ }^{1}$ It's different from the partition into MathGames and PlayGames defined in [7].
} 
Here we study an extension of Wythoff to four piles, which appears to be a PrimGame. The efforts in defining a "right" extension, and particularly in proving the validity of the winning strategy, are considerably greater than those for three-pile extensions. We present four winning strategies, algebraic, recursive, arithmetic and word-mapping. The recursive is the easiest to describe, though it seems to be hardest computationally. Actually it's also polynomial-time [12]. The algebraic depends on iterations of the floor function, the arithmetic on the Fibonacci numeration system and the word-mapping on a morphism-like mapping. All are polynomial time winning strategies.

The Flora game is a 2-player game played on 4 piles of tokens. We denote positions of Flora by $\left(a_{1}, a_{2}, a_{3}, a_{4}\right)$ with $0 \leq a_{1} \leq a_{2} \leq a_{3} \leq a_{4}$. It goes without saying that every pile must contain a nonnegative number of tokens at all times.

The end position is $T_{0}:=(0,0,0,0)$. The first player unable to move (because the present position is $T_{0}$ ) loses; the opponent wins.

There are 3 rules of move:

I. Any positive number of tokens from up to 3 piles may be removed.

II. From a nonzero position one can move to $T_{0}$ if any of the following 3 conditions hold: (i) two piles have the same size, (ii) $a_{3}-a_{2}=1$, (iii) $a_{1}=h(n$ ) and $a_{2}<h^{2}(n)-2$ for some $n \in \mathbb{Z}_{\geq 1}$.

III. If $0<a_{1}<a_{2}<a_{3}<a_{4}$, one can remove $p>0$ from $a_{3}, q>0$ from $a_{4}$ and an arbitrary nonnegative integer from $a_{1}$ and $a_{2}$, subject to:

(i) $q=p$ if $a_{4}-a_{3} \notin V_{2}$, except for the proviso that if $a_{3}-p$ is the second smallest component in the quadruple moved to, then $p \neq 5$.

(ii) $q=p+1$ if $a_{4}-a_{3} \in V_{2}$.

We say that a move in Flora is legal if it is consistent with the rules (I)-(III).

Note. If the position moved to under rule $\operatorname{III}(\mathrm{i})$ is $\left(b_{1}, b_{2}, b_{3}, b_{4}\right)$ (where of course $\left.0 \leq b_{1} \leq b_{2} \leq b_{3} \leq b_{4}\right)$, then $a_{3}-p=b_{i}, a_{4}-p=b_{j}$ for some $1 \leq i<j \leq 4$. Then $a_{4}-a_{3}=b_{j}-b_{i}=t$ for some $t \in \mathbb{Z}_{\geq 1}$, and normally $t \neq p$.

\subsection{Algebraic Formulation of the $P$-positions}

The set of $P$-positions of a game is the set of game positions from which the second (Previous) player can force a win. The set of all $P$-positions of a game is denoted $\mathcal{P}$. In particular, for Flora, $T_{0} \in \mathcal{P}$.

Let

$$
\begin{gathered}
A_{n}=h(n), \quad B_{n}=a^{3}(n), \quad C_{n}=h^{2}(n), \quad D_{n}=h^{3}(n), \\
A=\cup_{n=1}^{\infty} A_{n}, \quad B=\cup_{n=1}^{\infty} B_{n}, \quad C=\cup_{n=1}^{\infty} C_{n}, \quad D=\cup_{n=1}^{\infty} D_{n}, \\
T_{n}:=\left(A_{n}, B_{n}, C_{n}, D_{n}\right), \quad T=\cup_{n=0}^{\infty} T_{n} .
\end{gathered}
$$

A prefix of $T$ of size 19 is shown in Table 1 . We shall presently show that $T$ constitutes the set of $P$-positions of Flora. Assuming the truth of this assertion, we illustrate simple moves in instances of Flora. 
Examples. (i) From each of the positions $(4,6,9,9),(5,8,9,14),(7,8,11,20)$ one can move to $T_{0} \in \mathcal{P}$ by II.

(ii) From $(6,7,9,14)$, one can move to $(5,6,8,12) \in \mathcal{P}$ by I.

(iii) From $(19,21,22,32)$ can move as follows: $19 \rightarrow 9,21 \rightarrow 11,22 \rightarrow 7$, $32 \rightarrow 17$, resulting in $(7,9,11,17) \in \mathcal{P}$ by $\mathrm{III}(\mathrm{i})$.

(iv) From $(24,29,32,37)$, one can move to $(5,6,8,12) \in \mathcal{P}$ by III(ii) (since $\left.37-32=5 \in V_{2}\right)$.

Table 1: $P$-positions of Flora.

\begin{tabular}{c|cccc}
$n$ & $h(n)$ & $a^{3}(n)$ & $h^{2}(n)$ & $h^{3}(n)$ \\
\hline 0 & 0 & 0 & 0 & 0 \\
1 & 2 & 1 & 3 & 4 \\
2 & 5 & 6 & 8 & 12 \\
3 & 7 & 9 & 11 & 17 \\
4 & 10 & 14 & 16 & 25 \\
5 & 13 & 19 & 21 & 33 \\
6 & 15 & 22 & 24 & 38 \\
7 & 18 & 27 & 29 & 46 \\
8 & 20 & 30 & 32 & 51 \\
9 & 23 & 35 & 37 & 59 \\
10 & 26 & 40 & 42 & 67 \\
11 & 28 & 43 & 45 & 72 \\
12 & 31 & 48 & 50 & 80 \\
13 & 34 & 53 & 55 & 88 \\
14 & 36 & 56 & 58 & 93 \\
15 & 39 & 61 & 63 & 101 \\
16 & 41 & 64 & 66 & 106 \\
17 & 44 & 69 & 71 & 114 \\
18 & 47 & 74 & 76 & 122
\end{tabular}

Notation 3. For $n \in \mathbb{Z}_{>1}$, let $\Delta_{D C}(n):=h^{3}(n)-h^{2}(n), \Delta_{D B}(n):=h^{3}(n)-$ $a^{3}(n), \Delta_{D A}(n):=h^{3}(n)-h(n), \Delta_{C B}(n):=h^{2}(n)-a^{3}(n), \Delta_{C A}(n):=h^{2}(n)-$ $\left.h(n), \Delta_{B A}(n):=a^{(} n\right)-h(n), \Delta(n)=\Delta_{D C}(n) \cup \Delta_{D B}(n) \cup \Delta_{D A}(n) \cup \Delta_{C B}(n) \cup$ $\Delta_{C A}(n) \cup \Delta_{B A}(n), \Delta=\cup_{n=1}^{\infty} \Delta(n)$.

Lemma 4. (i) $\Delta_{D C}(n)=a^{2}(n)$,

(ii) $\Delta_{D B}(n)=a^{2}(n)+2$,

(iii) $\Delta_{D A}(n)=u^{2}(n)$,

(iv) $\Delta_{C B}(n)=2$,

(v) $\Delta_{C A}(n)=a(n)$,

(vi) $\Delta_{B A}(n)=a(n)-2$,

(vii) $\Delta=\mathbb{Z}_{>1} \backslash V_{2}$,

(viii) $\Delta=\cup_{n=1}^{\infty}\left(\Delta_{D C}(n) \cup \Delta_{D B}(n) \cup \Delta_{D A}(n)\right)$. 
Proof. (i) This is Theorem 1 (e1) for $k=2$.

(ii) Theorem 1 (e2) for $k=2$.

(iii) $\Delta_{D A}(n)=\left(h^{3}(n)-h^{2}(n)\right)+\left(h^{2}(n)-h(n)\right)=a^{2}(n)+a(n)=u^{2}(n)$ by Theorem 1 (e1) and (b).

(iv) Theorem 1 (c).

(v) Theorem 1 (e1).

(vi) $\Delta_{B A}(n):\left(a^{3}(n)-h^{2}(n)\right)+\left(h^{2}(n)-h(n)\right)=a(n)-2$.

(vii) Notice that for every $n \in \mathbb{Z}_{\geq 1}, \quad a^{2}(n) \in G_{1}, a^{2}(n)+2 \in G_{1}$ (by Theorem 1 (h1)), $2 \in U_{2}, a(n)-2 \in G_{1} \cup U_{2}$ (by (h3). It then follows from (iii) and (v) that $\Delta=G_{1} \cup U_{2}$. The result follows since the sets $G_{1}, U_{2}, V_{2}$ clearly partition $\mathbb{Z}_{>1}$.

(viii) Follows from (i)-(iii), (vii) and Theorem 1 (h2).

Lemma 5. For fixed $n \in \mathbb{Z}_{>1}$, let $0<t<a^{2}(n), t \notin V_{2}$. Then there exists $0 \leq m<n$ such that $t \in \Delta_{D C}(m) \cup \Delta_{D B}(m) \cup \Delta_{D A}(m)$.

Proof. We have $t<a^{2}(n)=\Delta_{D C}(n)<\Delta_{D B}(n)<\Delta_{D A}(n)$. It then follows from Lemma 4(viii), that there must be some $m<n$ for which $t \in \Delta_{D C}(m) \cup$ $\Delta_{D B}(m) \cup \Delta_{D A}(m)$.

Theorem 2. The set $T$ constitutes the set of P-positions of the game Flora.

Proof. To begin with we note the following facts:

- Lemma 1 implies that each of the sequences $A_{n}, B_{n}, C_{n}, D_{n}$ is increasing (Lemma 1).

- $A, B, C, D$ partition $\mathbb{Z}_{\geq 1}$ (Theorem 1 (a)).

It evidently suffices to prove the following two statements:

(A) Every move from any position in $T$ results in a position outside $T$.

(B) For every position outside $T$ there is a move into a position in $T$.

(A) Clearly there is no legal move $T_{1} \rightarrow T_{0}$. Suppose that there are positions $T_{n}, T_{m}$ with $m<n, n \geq 2$ such that there is a legal move $T_{n} \rightarrow T_{m}$. This move must be of type III, since $A, B, C, D$ partition $\mathbb{Z}_{>1}$, from which it follows easily, using Lemma 4, that $A_{n}<B_{n}<C_{n}<D_{n}$ for $n \geq 2$.

By Lemma 4(vii), $\Delta_{D C}(n) \notin V_{2}$, so we have to consider only move III(i). We first show that $D_{n}-p$ can only be $D_{m}$. It cannot be $A_{m}$, since then $C_{n}-p<A_{m}$ has no place in row $m$ of $T$. Suppose $D_{n}-p=B_{m}$. Then $C_{n}-p=A_{m}$. But $\Delta_{B A}(m)<\Delta_{B A}(n)=a(n)-2<\Delta_{D C}(n)=a^{2}(n)$, contradicting the move rule III(i). Suppose $D_{n}-p=C_{m}$. Since $C_{n}-B_{n}=2$ for all $n \in \mathbb{Z}_{\geq 1}$, we have $C_{n}-p=A_{m}$. But $\Delta_{C A}(m)=\Delta_{B A}(m)+2 \leq$ $\Delta_{B A}(n)+1=a(n)-1<\Delta_{D C}(n)=a^{2}(n)$, again contradicting move rule III(i). Thus indeed $D_{n}-p=D_{m}$.

Suppose $C_{n}-p=C_{m}$. Subtracting, $\Delta_{D C}(n)=\Delta_{D C}(m)$, so $a^{2}(n)=a^{2}(m)$ which is impossible for $m<n$ since the sequence $g^{2}(\ell)$ is strictly increasing. 
Suppose $C_{n}-p=B_{m}=C_{m}-2$. Then $\Delta_{D C}(n)=\Delta_{D B}(m)=\Delta_{D C}(m)+2$ which is possible if and only if $p=5$ and $m=n-1$. But this case is excluded by the proviso. Finally, suppose that $C_{n}-p=A_{m}$. Then $\Delta_{D C}(n)=\Delta_{D A}(m)$. By Lemma 4 this is equivalent to $a^{2}(n)=u^{2}(m)$. This is possible for no $m<n$ by disjointness.

(B) Let $\left(a_{1}, a_{2}, a_{3}, a_{4}\right) \notin T, 0 \leq a_{1} \leq a_{2} \leq a_{3} \leq a_{4}$. If there is equality in any of these or $a_{3}-a_{2}=1$, a move of type I or II leads to $T_{0}$. So we may assume $0<a_{1}<a_{2}<a_{2}+1<a_{3}<a_{4}$. By the complementarity of $A, B$, $C, D, a_{1}$ appears in precisely one component of precisely one $T_{n}, n \geq 1$. If $a_{1}=D_{n}$, move $a_{2} \rightarrow A_{n}, a_{3} \rightarrow B_{n}, a_{3} \rightarrow C_{n}$.

So suppose that $a_{1}=C_{n}$.

If $a_{4} \geq D_{n}$, move $a_{2} \rightarrow A_{n}, a_{3} \rightarrow B_{n}, a_{4} \rightarrow D_{n}$. So assume $a_{4}<D_{n}$. Let

$$
t:=a_{4}-a_{3}
$$

We consider 2 cases.

(a) $t \notin V_{2}$, and (b) $t \in V_{2}$.

(a) $t \notin V_{2}$. We have

$$
0<t=a_{4}-a_{3}<D_{n}-a_{3}<D_{n}-a_{1}=D_{n}-C_{n}=\Delta_{D C}(n)=a^{2}(n) .
$$

By Lemma 5, there exists $m<n$ such that either (i) $t=\Delta_{D C}(m)$, or (ii) $t=$ $\Delta_{D B}(m)$, or (iii) $t=\Delta_{D A}(m)$.

For case (i), move $\left(a_{1}, a_{2}, a_{3}, a_{4}\right) \rightarrow\left(A_{m}, B_{m}, C_{m}, D_{m}\right)$. This is a legal move: $a_{1}=C_{n}>A_{n}>A_{m}$, and $a_{2}>a_{1}=C_{n}>B_{n}>B_{m}, a_{3}>a_{1}=C_{n}>C_{m}$, $a_{4}=a_{3}+D_{m}-C_{m}>D_{m}$, so this move (as well as in the remainder of this proof) is of the form III.

For case (ii), move $a_{1} \rightarrow A_{m}, a_{2} \rightarrow C_{m}, a_{3} \rightarrow B_{m}, a_{4} \rightarrow D_{m}$. This is a legal move: $a_{1}=C_{n}>A_{n}>A_{m}, a_{2}>a_{1}=C_{n}>C_{m}, a_{3}>a_{1}=C_{n}>C_{m}>B_{m}$, $a_{4}=a_{3}+D_{m}-B_{m}>D_{m}$.

For case (iii), move $a_{1} \rightarrow B_{m}, a_{2} \rightarrow C_{m}, a_{3} \rightarrow A_{m}, a_{4} \rightarrow D_{m}$. This is a legal move: $a_{1}=C_{n}>B_{n}>B_{m}, a_{2}>a_{1}=C_{n}>C_{m}, a_{3}>a_{1}=C_{n}>C_{m}>A_{m}$, $a_{4}=a_{3}+D_{m}-A_{m}>D_{m}$.

(b) $t \in V_{2}$. To remind ourselves, $t=a_{4}-a_{3}$ and we have $a_{1}=C_{n}, a_{4}<$ $D_{n}$. Now $t-1 \in\left(V_{2}-1\right)$. Since $\left(V_{2}-1\right) \subset G_{2}$ (Theorem 1 (h4)), we have $t-1=a^{2}(m)$ for suitable $m \in \mathbb{Z}_{\geq 1}$. Also $\Delta_{D C}(m)=a^{2}(m)$ (Lemma 4 (i)). So we move: $\left(a_{1}, a_{2}, a_{3}, a_{4}\right) \rightarrow\left(A_{m}, B_{m}, C_{m}, D_{m}\right)$. This is a legal move:

- $m<n$, since $\Delta_{D C}(m)=a_{4}-a_{3}-1<D_{n}-a_{1}=D_{n}-C_{n}=\Delta_{D C}(n)$.

- $a_{1}=C_{n}>A_{n}>A_{m}, a_{2}>a_{1}=C_{n}>B_{n}>B_{m}, a_{3}>a_{1}=C_{n}>C_{m}$, $a_{4}=a_{3}+1+D_{m}-C_{m}>a_{1}+D_{m}-C_{m}=C_{n}+D_{m}-C_{m}>D_{m}$. 
So suppose that $a_{1}=B_{n}$.

If $a_{4} \geq D_{n}$, then move $a_{2} \rightarrow A_{n}, a_{3} \rightarrow C_{n}, a_{4} \rightarrow D_{n}$. This is a legal move, since $a_{2}>a_{1}=B_{n}>A_{n}$ and

$$
a_{3} \geq a_{2}+1 \geq a_{1}+2=B_{n}+2=C_{n} .
$$

Therefore we may assume $a_{4}<D_{n}$. The proof is similar to the above case $a_{1}=C_{n}$. We have $a_{3} \geq C_{n}$, and $0<t-1<t=a_{4}-a_{3}<D_{n}-a_{3} \leq \Delta_{D C}(n)$. Hence by Lemma 5 there is $m<n$ such that, for case (a), either (i) $t=\Delta_{D C}(m)$, or (ii) $t=\Delta_{D B}(m)$, or (iii) $t=\Delta_{D A}(m)$. For case (b) we have $t-1=a^{2}(m)$ for some $m \in \mathbb{Z}_{\geq 1}$.

(a) $t \notin V_{2}$.

For case (i), move $a_{2} \rightarrow A_{m},\left(a_{3}, a_{4}\right) \rightarrow\left(C_{m}, D_{m}\right)$. This is a legal move: $a_{2}>a_{1}=B_{n}>A_{n}>A_{m}, a_{3} \geq C_{n}>C_{m}$,

$$
a_{4}=a_{3}+D_{m}-C_{m} \geq C_{n}+D_{m}-C_{m}>D_{m} .
$$

For case (ii), move $a_{1} \rightarrow A_{m}, a_{2} \rightarrow C_{m}, a_{3} \rightarrow B_{m}, a_{4} \rightarrow D_{m}$. This is a legal move: $a_{1}=B_{n}>A_{n}>A_{m}, a_{2} \geq a_{1}+1=B_{n}+1=C_{n}-1 \geq C_{m}$, $a_{3}>a_{1}=B_{n}>B_{m}, a_{4}=a_{3}+D_{m}-B_{m} \geq C_{n}+D_{m}-B_{m}>D_{m}$.

For case (iii), move $a_{1} \rightarrow B_{m}, a_{2} \rightarrow C_{m}, a_{3} \rightarrow A_{m}, a_{4} \rightarrow D_{m}$. This is a legal move: $a_{1}=B_{n}>B_{m}, a_{2} \geq a_{1}+1=B_{n}+1=C_{n}-1 \geq C_{m}$, $a_{3}>a_{1}=B_{n}>B_{m}>A_{m}, a_{4}=a_{3}+D_{m}-A_{m}>D_{m}$.

(b) $t \in V_{2}$. We have $t=a_{4}-a_{3}, a_{1}=B_{n}, a_{4}<D_{n}$. As in case (b) above, we move $\left(a_{1}, a_{2}, a_{3}, a_{4}\right) \rightarrow\left(A_{m}, B_{m}, C_{m}, D_{m}\right)$. This is a legal move:

- $m<n$, since $\Delta_{D C}(m)=a_{4}-a_{3}-1<D_{n}-a_{3}-1 \leq D_{n}-C_{n}-1<\Delta_{D C}(n)$.

- $a_{1}=B_{n}>A_{n}>A_{m}, a_{2}>a_{1}=B_{n}>B_{m}, a_{3} \geq C_{n}>C_{m}, a_{4}=$ $a_{3}+1+D_{m}-C_{m} \geq C_{n}+1+D_{m}-C_{m}>D_{m}$.

Finally, we consider the case $a_{1}=A_{n}=h(n)$.

If $a_{2}<h^{2}(n)-2$ we can move to $T_{0}$ (rule (II)(iii)). Otherwise, $a_{2} \geq$ $h^{2}(n)-2=B_{n}$. Since $a_{3}-a_{2}>1$, we have $a_{3} \geq B_{n}+2=C_{n}$. If $a_{4} \geq D_{n}$, then at least one of the inequalities for $a_{2}, a_{3}, a_{4}$ is strict, since $\left(a_{1}, a_{2}, a_{3}, a_{4}\right) \notin T$. Then move $\left(a_{1}, a_{2}, a_{3}, a_{4}\right) \rightarrow\left(A_{n}, B_{n}, C_{n}, D_{n}\right)$. If $a_{4}<D_{n}$ then for case (a) there is $m<n$ such that $0<t=a_{4}-a_{3}<\Delta_{D C}(n)$. Hence by Lemma 5 , there is $m<n$ such that either (i) $t=\Delta_{D C}(m)$, or (ii) $t=\Delta_{D B}(m)$, or (iii) $t=\Delta_{D A}(m)$. For case (b), $0<t-1=a^{2}(m)$ for some $m \in \mathbb{Z}_{\geq 1}$.

(a) $t \notin V_{2}$. For case (i) move $\left(a_{1}, a_{2}, a_{3}, a_{4}\right) \rightarrow\left(A_{m}, B_{m}, C_{m}, D_{m}\right)$. This is a legal move, since $m<n$ implies $a_{1}=A_{n}>A_{m}, a_{2} \geq B_{n}>B_{m}, a_{3} \geq C_{n}>C_{m}$, $D_{n}>D_{m}$. For case (ii), move $a_{1} \rightarrow A_{m}, a_{2} \rightarrow C_{m}, a_{3} \rightarrow B_{m}, a_{4} \rightarrow D_{m}$. This is a legal move: $a_{1}=A_{n}>A_{m}, a_{2} \geq B_{n}=C_{n}-2>C_{m}$, where the strict inequality follows since $C_{n}-C_{m} \geq 3$ (Theorem 1 (f4) for $k=3$ ). Also $a_{3} \geq C_{n}>B_{n}>B_{m}, a_{4}=a_{3}+D_{m}-B_{m}>D_{m}$. For case (iii) move $a_{1} \rightarrow B_{m}$, 
$a_{2} \rightarrow C_{m}, a_{3} \rightarrow A_{m}, a_{4} \rightarrow D_{m}$. We have to prove the legality of this move. We begin by showing that $a_{1}=A_{n}>B_{m}$. Notice that

$$
\begin{aligned}
t & =a_{4}-a_{3}=\Delta_{D A}(m)=u^{2}(m) \quad(\text { Lemma } 4) \\
& <\Delta_{D C}(n)=a^{2}(n) \quad(\text { Lemma } 4) \\
& =h(n)-1 \quad(\text { Theorem } 1 \text { (c)) }
\end{aligned}
$$

Thus $h(n)>u^{2}(m)+1$. But $h(n)=A_{n}$ and $u^{2}(m)=a^{3}(m)+1$ (by Theorem 1 (d) $)=B_{m}+1$, so indeed $A_{n}>B_{m}+2>B_{m}$. Next,

$$
\begin{aligned}
a_{2} & \geq B_{n}=a^{3}(n)=h^{2}(n)-2 \quad(\text { Theorem } 1(\mathbf{d})) \\
& >h^{2}(n-1) \quad(\text { Theorem } 1(\mathbf{f} 4))=C_{n-1} \geq C_{m} .
\end{aligned}
$$

Also $a_{3} \geq C_{n}>C_{m}>A_{m}$ and $a_{4}=a_{3}+D_{m}-A_{m} \geq C_{n}+D_{m}-A_{m}>D_{m}$.

(b) $t \in V_{2}$. We have $t=a_{4}-a_{3}, a_{1}=A_{n}, a_{4}<D_{n}, t-1=a^{2}(m)=\Delta_{D C}(m)$ for some $m \in \mathbb{Z}_{\geq 1}$. As above we move $\left(a_{1}, a_{2}, a_{3}, a_{4}\right) \rightarrow\left(A_{m}, B_{m}, C_{m}, D_{m}\right)$. This is a legal move:

- $m<n$, since $\Delta_{D C}(m)=a_{4}-a_{3}-1<D_{n}-C_{n}-1<\Delta_{D C}(n)$.

- $a_{1}=A_{n}>A_{m}, a_{2} \geq B_{n}>B_{m}, a_{3} \geq C_{n}>C_{m}, a_{4}=a_{3}+1+D_{m}-C_{m}>$ $C_{n}+D_{m}-C_{m}>D_{m}$

Theorem 3. The algebraic winning strategy of Flora precipitates a polynomialtime algorithm for consummating a win.

Proof. Given a position $\left(a_{1}, a_{2}, a_{3}, a_{4}\right)$ of Flora with $0 \leq a_{1} \leq a_{2} \leq a_{3} \leq a_{4}$. Its input size is $O\left(\log a_{1}+\log a_{2}+\log a_{3}+\log a_{4}\right)$. Whether or not move rules II(i) or II(ii) apply can be checked trivially. We know (Theorem 1 (a)) that $a_{1}$ is precisely one of $a^{3}(n), h^{3}(n), h^{2}(n), h(n)$. We have to find out which it is, and the corresponding value of $n$.

Suppose first that $a_{1}=a^{3}(n)=\lfloor\varphi\lfloor\varphi\lfloor n \varphi\rfloor\rfloor\rfloor$. Using the inequality $x-$ $1<\lfloor x\rfloor \leq x$, a straightforward computation shows that $\left\lfloor a_{1} \varphi^{-3}\right\rfloor+1 \leq n \leq$ $\left\lfloor\left(a_{1}+1\right) \varphi^{-3}\right\rfloor+1$. Computing $\varphi$ to $O\left(\log a_{1}\right)$ places gives the range for the candidate values of $n$ and for each of them (one or two), we have to compute $a^{3}(n)$, comparing it with $a_{1}$. A similar computation can be done for $h^{3}(n)$, $h^{2}(n), h(n)$. (Notice that there is not necessarily an integer candidate $n$ for some of these ranges. For example, if we suppose that $a_{1}=h^{3}(n)$, then we get $\left\lfloor a_{1} \varphi^{-4}\right\rfloor+1 \leq n \leq\left\lfloor a_{1} \varphi^{-4}+2 \varphi^{-2}\right\rfloor$.) The same method also indicates whether or not $a_{4}-a_{3} \in V_{2}$, or whether move rule II(iii) applies. All these computations can be done in linear time in the input size.

Finally, we use a binary search to find $m \in[0, n]$ such that if $a_{4}-a_{3} \notin V_{2}$, then $h^{3}(m)-h^{2}(m)=a_{4}-a_{3}$ or some other difference of the columns in the $m$-th row is $a_{4}-a_{3}$. Similarly for the case $a_{4}-a_{3} \in V_{2}$. 


\subsection{Recursive Formulation of the $P$-positions}

Let $S \varsubsetneqq \mathbb{Z}_{\geq 1}$ and $\bar{S}=\mathbb{Z}_{\geq 1} \backslash S$. The "Minimum EXcludant" of $S$ is defined by

$$
\operatorname{mex} S=\min \bar{S}=\text { least positive integer not in } \mathrm{S} \text {. }
$$

In particular, the mex of the empty set is 1. (This somewhat nonstandard definition of the mex function is needed for $\S 5$.)

Let $T_{0}^{\prime}=(0,0,0,0), T_{1}^{\prime}=(2,1,3,4)$. If $T_{m}^{\prime}:=\left(A_{m}^{\prime}, B_{m}^{\prime}, C_{m}^{\prime}, D_{m}^{\prime}\right)$ has already been defined for all $m<n \quad(n \geq 2)$, then let

$$
\begin{gathered}
A_{n}^{\prime}=\operatorname{mex}\left\{A_{i}^{\prime}, B_{i}^{\prime}, C_{i}^{\prime}, D_{i}^{\prime}: 0 \leq i<n\right\}, \\
B_{n}^{\prime}=\left\{\begin{array}{cc}
B_{n-1}^{\prime}+3 & \text { if } A_{n}^{\prime}-A_{n-1}^{\prime}=2 \\
B_{n-1}^{\prime}+5 & \text { otherwise, }
\end{array}\right. \\
C_{n}^{\prime}=B_{n}^{\prime}+2, \\
D_{n}^{\prime}=\left\{\begin{array}{ccc}
D_{n-1}^{\prime}+5 & \text { if } A_{n}^{\prime}-A_{n-1}^{\prime}=2 \\
D_{n-1}^{\prime}+8 & \text { otherwise. }
\end{array}\right.
\end{gathered}
$$

Let $T^{\prime}:=\cup_{n=0}^{\infty} T_{n}^{\prime}$.

Theorem 4. The set $T^{\prime}$ constitutes the set of P-positions of the game Flora.

Proof. We show that for all $m \in \mathbb{Z}_{\geq 0}, A_{m}^{\prime}=A_{m}, B_{m}^{\prime}=B_{m}, C_{m}^{\prime}=C_{m}, D_{m}^{\prime}=$ $D_{m}$. Suppose this holds for all $m<n \quad(n \geq 1)$. Let $E=\operatorname{mex}\left\{A_{i}, B_{i}, C_{i}, D_{i}\right.$ : $0 \leq i<n\}$. The value $E$ cannot have been assumed in any of the four sequences for $m<n$, since $A, B, C, D$ split $\mathbb{Z}_{\geq 1}$, so $E \geq A_{n}$. If $E>A_{n}$, then $A_{n}$ would never be assumed since the sequences are strictly increasing, again contradicting the complementarity of the sequences. Thus $A_{n}=E=A_{n}^{\prime}$, and the other 3 equalities follow from Theorem 2 (f).

The definition of the set $T^{\prime}$ is straightforward; it doesn't use the functions $h(n), a(n)$ used for defining $T$. Thus the recursive computation of $T^{\prime}$ looks easier than that of the set $T$. Moreover, the proof of Theorem 4 is very short, and that of Theorem 2 is long.

However, the proof of Theorem 4 leans heavily on Theorems 2 and 1 . If the initial position of the game is $\left(a_{1}, a_{2}, a_{3}, a_{4}\right)$, the input size is $\log a_{1}+$ $\log a_{2}+\log a_{3}+\log a_{4}$. The time needed to compute whether the position is a $P$-position or not, seems to be proportional to $a_{1}+a_{2}+a_{3}+a_{4}$, because the unwieldy mex function appears to require scanning previous entries of the sequences $A_{n}, B_{n}, C_{n}, D_{n}$. However, a new method [12] shows that actually also the algorithm implied by Theorem 4 is polynomial. 


\subsection{Arithmetic Formulation of the $P$-positions}

For $N \in \mathbb{Z}_{\geq 1}$, let $R(N)=\left(d_{m}, \ldots, d_{0}\right)$ be the representation of $N$ in the Fibonacci numeration system (recall Notation 2$)$. Then $\left(d_{m}, \ldots, d_{0}, 0\right)$ is the left shift of $R(N)$.

Theorem 5. $R(A)$ is the set of all representations that end in an odd number of 0-bits in the Fibonacci numeration system, $R(B)$ the set of all representations that end in $001, R(C)$ the set of all representations that end in a positive even number of 0-bits, and $R(D)$ the set of all representations that end in 101 . Moreover, for every $n \in \mathbb{Z}_{\geq 1}, R\left(C_{n}\right)$ is the left shift of $R\left(A_{n}\right)$.

See Table 3.3 for an example.

Table 3.3: Representation of the $P$-positions in the Fibonacci numeration system.

\begin{tabular}{rrrrrrr|rrr|rrrrrrrr}
21 & 13 & 8 & 5 & 3 & 2 & 1 & $A_{n}$ & $n$ & $B_{n}$ & 34 & 21 & 13 & 8 & 5 & 3 & 2 & 1 \\
\hline & & & & & 1 & 0 & 2 & 1 & 1 & & & & & & & & 1 \\
& & & 1 & 0 & 0 & 0 & 5 & 2 & 6 & & & & & 1 & 0 & 0 & 1 \\
& & & 1 & 0 & 1 & 0 & 7 & 3 & 9 & & & & 1 & 0 & 0 & 0 & 1 \\
& & 1 & 0 & 0 & 1 & 0 & 10 & 4 & 14 & & & 1 & 0 & 0 & 0 & 0 & 1 \\
& 1 & 0 & 0 & 0 & 0 & 0 & 13 & 5 & 19 & & & 1 & 0 & 1 & 0 & 0 & 1 \\
& 1 & 0 & 0 & 0 & 1 & 0 & 15 & 6 & 22 & & 1 & 0 & 0 & 0 & 0 & 0 & 1 \\
& 1 & 0 & 1 & 0 & 0 & 0 & 18 & 7 & 27 & & 1 & 0 & 0 & 1 & 0 & 0 & 1 \\
& 1 & 0 & 1 & 0 & 1 & 0 & 20 & 8 & 30 & & 1 & 0 & 1 & 0 & 0 & 0 & 1 \\
1 & 0 & 0 & 0 & 0 & 1 & 0 & 23 & 9 & 35 & 1 & 0 & 0 & 0 & 0 & 0 & 0 & 1 \\
1 & 0 & 0 & 1 & 0 & 0 & 0 & 26 & 10 & 40 & 1 & 0 & 0 & 0 & 1 & 0 & 0 & 1
\end{tabular}

\begin{tabular}{rrrrrrrr|rrr|rrrrrrrrr}
34 & 21 & 13 & 8 & 5 & 3 & 2 & 1 & $C_{n}$ & $n$ & $D_{n}$ & 55 & 34 & 21 & 13 & 8 & 5 & 3 & 2 & 1 \\
\hline & & & & & 1 & 0 & 0 & 3 & 1 & 4 & & & & & & & 1 & 0 & 1 \\
& & & 1 & 0 & 0 & 0 & 0 & 8 & 2 & 12 & & & & & 1 & 0 & 1 & 0 & 1 \\
& & & 1 & 0 & 1 & 0 & 0 & 11 & 3 & 17 & & & & 1 & 0 & 0 & 1 & 0 & 1 \\
& & 1 & 0 & 0 & 1 & 0 & 0 & 16 & 4 & 25 & & & 1 & 0 & 0 & 0 & 1 & 0 & 1 \\
& 1 & 0 & 0 & 0 & 0 & 0 & 0 & 21 & 5 & 33 & & & 1 & 0 & 1 & 0 & 1 & 0 & 1 \\
& 1 & 0 & 0 & 0 & 1 & 0 & 0 & 24 & 6 & 38 & & 1 & 0 & 0 & 0 & 0 & 1 & 0 & 1 \\
& 1 & 0 & 1 & 0 & 0 & 0 & 0 & 29 & 7 & 46 & & 1 & 0 & 0 & 1 & 0 & 1 & 0 & 1 \\
& 1 & 0 & 1 & 0 & 1 & 0 & 0 & 32 & 8 & 51 & & 1 & 0 & 1 & 0 & 0 & 1 & 0 & 1 \\
1 & 0 & 0 & 0 & 0 & 1 & 0 & 0 & 37 & 9 & 59 & 1 & 0 & 0 & 0 & 0 & 0 & 1 & 0 & 1 \\
1 & 0 & 0 & 1 & 0 & 0 & 0 & 0 & 42 & 10 & 67 & 1 & 0 & 0 & 0 & 1 & 0 & 1 & 0 & 1
\end{tabular}

Proof. The proof is similar to that of Theorem 3 of [8]. For every $m \in \mathbb{Z}_{\geq 1}$, $R(\lfloor m \varphi\rfloor)$ ends in an even number of 0-bits (including 0 0-bits), and $R\left(\left\lfloor m \varphi^{\overline{2}}\right\rfloor\right)$ 
ends in an odd number of 0-bits $([5], \S 4)$. Hence $R(A)$ is the set of all numbers that end in an odd number of 0-bits in the Fibonacci numeration system, whereas each of the other 3 representations ends in an even number of 0-bits. Now $R(C)$ is the set of all numbers that end in a positive even number of 0-bits ([8]), hence $R(B)$ and $R(D)$ each end in a 1-bit. Recall that $C_{n}=B_{n}+2$. If $R(B)$ would contain a number with representation ending in 101 , then adding 2 to it would end in 1 (since $2+3=5$ is the next Fibonacci number), contradicting the form of $R\left(C_{n}\right)$. Therefore $R(B)$ is the set of all numbers ending in 001. By complementarity, $R(D)$ is therefore the set of all numbers ending in 101.

Since $R(A)$ is the set of all representations ending in an odd number of 0 bits, and $R(C)$ is the set of all representations ending in a positive even number of 0-bits, the latter is the left shift of the former. Suppose that $R\left(C_{m}\right)$ is the left shift of $R\left(A_{m}\right)$ for every $m<n$. If $R\left(C_{n}\right)$ would not be the left shift of $R\left(A_{n}\right)$, then it would be assumed later on (by complementarity), contradicting the strict increase of $C$.

This formulation of the $P$-positions is also easily seen to lead to a polynomialtime winning strategy.

\section{The Word-mapping Approach}

In this section we show how to construct $G_{k}$ recursively by a word-map for every $k \in \mathbb{Z}_{\geq 1}$. Similar methods can be used to construct other functions defined in $\S 2$. We also present our fourth, word-mapping, formulation of the $P$-positions of the game Flora. The length of any (sub)word $w$ is denoted by $|w|$.

\subsection{Word-mapping for $G_{k}$}

Define the morphism $1 \rightarrow 10,0 \rightarrow 1$. Its fixed point is the word: $F=$ $1011010110110 \ldots$, also known as the Fibonacci word. For $k \geq 1$, the characteristic function $\chi_{k}$ of $G_{k}$ is defined by

$$
\chi_{k}(m)=\left\{\begin{array}{l}
1 \text { if } \exists n \text { s.t. } \quad a^{k}(n)=m \\
0 \text { otherwise. }
\end{array}\right.
$$

Definition 1. Given a binary word $W$. A run of 0 s is any (possibly empty) subword of $W$ consisting solely of 0 s, flanked on the left and right by a 1-bit. A block is any subword consisting of a 1-bit followed by a run of 0 s.

Theorem 6. For every $k \geq 1$, the word-mapping for producing the characteristic function $\chi_{k}$ of $G_{k}$, beginning with $10^{F_{k-1}-1}$, is:

$$
10^{F_{k-1}-1} \rightarrow 10^{F_{k}-1}, \quad 0^{F_{k-2}} \rightarrow 10^{F_{k-1}-1} .
$$

Proof. Notice that for $G_{1}$, the word-mapping is simply the well-known morphism $1 \rightarrow 10,0 \rightarrow 1$, which produces $F$. Moreover, $\chi_{1}=F$. See e.g., [1], ch. 9 and $[11]$. 
The word-map is well-defined. Indeed, the initial block of length $F_{k-1}$ is mapped into a block $B_{1}$ of length $F_{k}$. In the second iteration, the prefix of length $F_{k-1}$ of $B_{1}$ is again mapped into $B_{1}$. The remaining abutting suffix of $B_{1}$ consists of $F_{k-2} 0 \mathrm{~s}$, so it is mapped into a block $B_{2}$ of length $F_{k-1}$. In the third iteration, $B_{1}$ and $B_{2}$ are generated again, and then the block $B_{2}$ of length $F_{k-1}$ generates a block $B_{1}$. Thus for all subsequent iterations only blocks of the form $B_{1}$ and $B_{2}$ are generated, and there is never any parsing conflict.

Since $\chi_{1}=1011010110110 \ldots$, Theorem $1(\mathbf{f})$ implies that $\chi_{2}=10010100100$ $1010010100 \ldots$, where we inserted into $\chi_{1} F_{1}-F_{0}=1$ zero to each run of $F_{0}-1=0$ zeros (i.e., one 0 between every consecutive $1 \mathrm{~s}$ ), and $F_{2}-F_{1}=1$ zero to each run of $F_{1}-1=1$ zeros. Doing this yields distances between consecutive $1 \mathrm{~s}$ in $\chi_{2}$ of $F_{1}$ and $F_{2}$, precisely at the locations where the distances between consecutive 1 s of $\chi_{1}$ are $F_{0}$ and $F_{1}$ respectively. Similarly, $\chi_{3}=1000010010000100001001000010010000 \ldots$, where we inserted into $\chi_{2}$ $F_{2}-F_{1}=1$ zero to each run of $F_{1}-1=1$ zero, and $F_{3}-F_{2}=2$ zeros to each run of $F_{2}-1=2$ zeros.

In general, for producing $\chi_{k+1}$ from $\chi_{k}$, we add to $\chi_{k} F_{k}-F_{k-1}=F_{k-2}$ zeros to each run of $F_{k-1}-1$ zeros and $F_{k+1}-F_{k}=F_{k-1}$ zeros to each run of $F_{k}$ zeros. This yields blocks of sizes $F_{k}$ and $F_{k+1}$ respectively, at the locations specified by Theorem 1(f).

Assume inductively that the word-mapping

$$
10^{F_{k-1}-1} \rightarrow 10^{F_{k}-1}, \quad 0^{F_{k-2}} \rightarrow 10^{F_{k-1}-1}
$$

produces $\chi_{k}$, so it generates distances between consecutive 1 s of $F_{k-1}$ and $F_{k}$ at the locations specified by Theorem $1(\mathbf{f})$. Then the word-mapping

$$
10^{F_{k}-1} \rightarrow 10^{F_{k+1}-1}, \quad 0^{F_{k-1}} \rightarrow 10^{F_{k}-1}
$$

produces $\chi_{k+1}$, since it adds $F_{k+1}-F_{k}$ to the $F_{k}$ 0s of the long 0-runs of $\chi_{k}$, and $F_{k}-F_{k-1} 0$ s to the $F_{k-1}$ short 0-runs of $\chi_{k}$.

\subsection{Word-mapping Formulation of of the $P$-positions}

Denote terms of $A_{n}, B_{n}, C_{n}, D_{n}$ by $a, b, c, d$ respectively.

Theorem 7. The word-mapping

$$
b a c \rightarrow b a c d a, \quad d a \rightarrow b a c,
$$

beginning with bac, generates the characteristic function of the P-positions of the Flora game.

Proof. The proof is rather similar to that of Theorem 6, and is therefore omitted.

This theorem also leads to a polynomial-time winning strategy, since induction shows that for every $k \in \mathbb{Z}_{\geq 1}$, the $k$-th application of the word-mapping generates a word of length $F_{k+2}$. 
Notice that if we replace bac by 1 and $d a$ by 0 , we get back our old morphic friend $1 \rightarrow 10,0 \rightarrow 1$.

\subsection{Characterization of the Sequences $G, H$, by the Fi- bonacci Numeration System}

We know from Lemma II and $\S 3.3$, that $R(a(n))$ ends in an even number of $0 \mathrm{~s}$, $R(h(n))$ in an odd number of $0 \mathrm{~s}, R\left(h^{2}(n)\right)$ in an even positive number of $0 \mathrm{~s}$, $R\left(h^{3}(n)\right)$ in 101 , and $R\left(g^{3}(n)\right)$ in $10^{s} 1, s \geq 2$. What's the general pattern?

Theorem 8. (i) $R\left(G_{1}\right)$ is the set of all representations that end in an even number of $0 s, R\left(H_{1}\right)$ is the set of all representations that end in an odd number of $0 s, R\left(G_{2}\right)$ is the set of all representations that end in a 1-bit, and $R\left(H_{2}\right)$ is the set of all representations that end in an even positive number of 0 s.

(ii) For every $n \in \mathbb{Z}_{\geq 1}, R(h(n))$ is the left shift of $R(a(n))$; and $R\left(h^{2}(n)\right)$ is the left shift of $R(h(n))$.

(iii) For every $k \in \mathbb{Z}_{\geq 3}$ and all $n \in \mathbb{Z}_{\geq 1}, R\left(G_{k}\right)$ is the set of all representations that end in the word $10^{s} 1$ for all $s \geq k-1$, and $R\left(H_{k}\right)$ is the set of all representations that end in the word $10^{k-2} 1$ (left 1-bit in position $k-1$ ).

Proof. Items (i) and (ii) are already known from Theorem 5 and Lemma II, and are included here only for the sake of completeness. We only have to point out the statement about $R\left(G_{2}\right)$, which follows from the fact that $G_{2}, H_{2}, H_{1}$ split the positive integers (see also [8], Theorem 3 ).

(iii) Induction on $k$. The base case $k=3$ was proved in Theorem 5. For $k \geq 3$, suppose that we already proved that $R\left(G_{k}\right)$ is the set of all representations that end in $10^{s} 1$ for all $s \geq k-1$, and $R\left(H_{k}\right)$ is the set of all representations that end in $10^{k-2} 1$. It clearly remains only to show that $R\left(G_{k+1}\right)$ is the set of all representations that end in $10^{s} 1$ for all $s \geq k$, and $R\left(H_{k+1}\right)$ is the set of all representations that end in the word $10^{k-1} 1$. Recall Theorem 1 (c): $h^{k}(n)=g^{k+1}(n)+F_{k-1}$. If $R\left(G_{k+1}\right)$ would contain a number, say $a^{k+1}(n)$, with representation ending in $10^{k-1} 1$ (with leftmost 1-bit in position $k$ ), then adding $F_{k-1}$ to it would result in a word with representation ending in $0^{k} 1$ because $F_{k-1}+F_{k}=F_{k+1}$ is the next Fibonacci number. But then $R\left(h^{k}(n)\right)$ would end in $10^{s} 1$ for some $s \geq k$, contradicting the induction hypothesis. Thus $R\left(H_{k+1}\right)$ is the set of all representations that end in the word $10^{k-1} 1$. Since $G_{k+1}, H_{k+1}, H_{k}, \ldots, H_{2}, H_{1}$ split the integers, $R\left(H_{k+1}\right)$ is the set of all representations that end in the word $10^{k-1} 1$.

Notes. (1) The general pattern of the representation of the suffixes of $H_{k}$ for $k \geq 3$ is quite different from that of $H_{1}$ and $H_{2}$, and both of these are different from each other. The same holds for $G_{k}, k \geq 3$ and $G_{1}$ and $G_{2}$. Therefore the induction proof could not have begun with $k=1$ or 2 .

(2) The statement in (i) about $R\left(G_{1}\right)$ and $R\left(H_{1}\right)$ is Theorem 9.1 .15 (see also Corollary 9.1.14) in [1], credited there to [10]. (It is also Lemma II above.) The proof method of [1] follows [3]. 
Membership Problem. Fix some $k \in \mathbb{Z}_{\geq 1}$. Given $N \in \mathbb{Z}_{\geq 1}$. Then $N$ is in precisely one of $G_{k}, H_{k}, H_{k-1}, \ldots, H_{2}, H_{1}$ (Theorem 1 (a)). Can the following problem be solved in polynomial time?

Problem. Determine the set in which $N$ lies.

Corollary 1. For every $k \in \mathbb{Z}_{\geq 1}$, the membership problem can be solved in linear time.

Proof. This can be proved by generalizing the method for computing $n$ in the proof of Theorem 3 to the case of arbitrary $k$. But a more "elegant" method is to compute the Fibonacci representation of $N$, which can be done in linear time in the input size $\Theta(\log N)$. Theorem 8 then implies that the membership problem can be solved by scanning the suffix of $R(N)$, at most all of its $\Theta(\log N)$ bits.

\section{$5 \quad$ Infinite Complementary Arrays}

The doubly infinite Stolarsky Array $A$ with entries $A(i, j), i, j \geq 1$ [22], is defined as follows: For every $m \geq 1, A(m, 1)=\operatorname{mex}\{A(i, j): i<m, j \geq 1\}$, $A(m, 2)=\lfloor\varphi A(m, 1)+1 / 2\rfloor$, and for all $i \geq 1, j \geq 3, A(i, j), A(i, j)=$ $A(i, j-1)+A(i, j-2)$. Then every positive integer appears precisely once in $A$. A beginning portion is exhibited in Table 2. Many variations, interspersions and dispersions have since been given, see e.g., [16], [19]. All are doubly infinite, $\lim _{j \rightarrow \infty}(A(i, j+1)-A(i, j))=\infty$ for every $i \geq 1$, and every positive integer appears precisely once in $A$.

Table 2: The Stolarsky array

$\begin{array}{rrrrrrl}1 & 2 & 3 & 5 & 8 & 13 & \ldots \\ 4 & 6 & 10 & 16 & 26 & 42 & \ldots \\ 7 & 11 & 18 & 29 & 47 & 76 & \ldots \\ 9 & 15 & 24 & 39 & 63 & 102 & \ldots \\ \vdots & \vdots & \vdots & \vdots & \vdots & \vdots & \end{array}$

For every $k \geq 1$, define the Flora-Array $L_{K}$ with the $k+1$ rows $H_{1}, H_{2}, \ldots, H_{k}, G_{k}$. This array has a different character. By Theorem 1(a), this singly-infinite array also has the property that every positive integer appears precisely once. Moreover, $A(i, j+1)-A(i, j) \in\left\{F_{i}, F_{i+1}\right\}$ is bounded for every fixed $i$ and all $j \geq 1$, but $\lim _{i \rightarrow \infty}(A(i, j+1)-A(i, j))=\infty$. Table 3 depicts the case $k=6$. The two bottom lines, below the horizontal line, illustrate the fact that $G_{7}, H_{7}$ split $G_{6}$, so replacing $G_{6}$ by $H_{7}$ and $G_{7}$ constitutes $L_{7}$. 
Table 3: A complementary Flora array $L_{6}$ with 7 rows

\begin{tabular}{c|rrrrrrrrrrrrr}
$n$ & 1 & 2 & 3 & 4 & 5 & 6 & 7 & 8 & 9 & 10 & 11 & 12 & 13 \\
\hline$H_{1}$ & 2 & 5 & 7 & 10 & 13 & 15 & 18 & 20 & 23 & 26 & 28 & 31 & 34 \\
$H_{2}$ & 3 & 8 & 11 & 16 & 21 & 24 & 29 & 32 & 37 & 42 & 45 & 50 & 55 \\
$H_{3}$ & 4 & 12 & 17 & 25 & 33 & 38 & 46 & 51 & 59 & 67 & 72 & 80 & 88 \\
$H_{4}$ & 6 & 19 & 27 & 40 & 53 & 61 & 74 & 82 & 95 & 108 & 116 & 129 & 142 \\
$H_{5}$ & 9 & 30 & 43 & 64 & 85 & 98 & 119 & 132 & 153 & 174 & 187 & 208 & 229 \\
$H_{6}$ & 14 & 48 & 69 & 103 & 137 & 158 & 192 & 213 & 247 & 281 & 302 & 336 & 370 \\
$G_{6}$ & 1 & 22 & 35 & 56 & 77 & 90 & 111 & 124 & 145 & 166 & 179 & 200 & 221 \\
\hline$H_{7}$ & 22 & 77 & 111 & 166 & 221 & 255 & 310 & 344 & $\cdots$ & & & & \\
$G_{7}$ & 1 & 35 & 56 & 90 & 124 & 145 & 179 & 200 & 234 & 268 & 289 & 323 & $\ldots$
\end{tabular}

\section{Epilogue}

We have generated sequences consisting of nested arbitrary applications of the floor function to $\varphi$ and $\varphi^{2}$, established many identities and relationships involving them and then applied them to formulate an algebraic winning strategy to the game Flora. We also presented recursive, arithmetic and word-mapping formulations of the winning strategy. In addition, we characterized the main sequences by means of the Fibonacci numeration system, and generated infinite complementary arrays of the sequences.

Can some of the relationships of the iterated floor functions be generalized to reals other than $\varphi$ and $\varphi^{2}$ ? As a first step, we could interchange $\varphi$ with $\varphi^{2}$, studying the ensuing sequences and the games implied by them. Specifically, define $g^{\prime}(n)=\left\lfloor n \varphi^{2}\right\rfloor, h^{\prime}(n)=\lfloor n \varphi\rfloor$, and for $k \geq 2, g^{\prime k}(n)=\left\lfloor\varphi^{2} g^{\prime k-1}(n)\right\rfloor$, $h^{\prime k}(n)=\left\lfloor\varphi^{2} g^{\prime k-1}(n)\right\rfloor$. If we define $G_{i}^{\prime}, H_{i}^{\prime}$ in the obvious way, it's straightforward to see that then the first item of Theorem 1, is preserved, namely $G_{k}^{\prime}, H_{k}^{\prime}, H_{k-1}^{\prime}, \ldots, H_{2}^{\prime}, H_{1}^{\prime}$ partition $\mathbb{Z}_{\geq 1}$. What games can be spawned from this partition?

More generally, it would be well to investigate which of the above results hold for which classes of positive reals beyond $\varphi$. For example, Lemma 1 and Theorem 1(a) clearly hold if we replace $\varphi$ by any irrational $\alpha \in(1,2)$ and $\varphi^{2}$ by $\beta=\alpha /(\alpha-1)$. Perhaps large parts of Theorem 1 can be generalized for the case where $\alpha=\left(2-t+\sqrt{t^{2}+4}\right) / 2, \beta=\alpha+t$, where $t$ is any given positive integer, since then the simple continued fraction of $\alpha$ is $[1, t, t, t, \ldots]$, so the numeration system arguments used in the proof of (c) and (d) of Theorem 1 carry over in a simple way. What games are induced by these relationships?

The notion of arbitrary iterations of the floor function appeared in [21] and [15]. In the former, the iterations are with rational numbers whose sizes depend on the iteration depth; in the latter, the aim is to represent the positive integers in the form of iterated floor functions involving $\varphi$ and $\varphi^{2}$. These are quite different from our iterations of the floor function. However, in [18] iterations of the form considered here were studied as pointed out above. See also [17]. 
The Raleigh game [8] is a 3-pile extension - not generalization - of Wythoff's game. Flora is an extension of Raleigh. Although Flora appears not to be decomposable into sums of more elementary games, we were able to formulate for it three polynomial-time winning strategies. The one based on the Fibonacci numeration system is of particular interest. It demonstrates once again that numeration systems can make strategies of games in PrimGame efficient, similarly to appropriate data structures - see [20].

We can also define a 5-pile extension of Flora, but in the sequence of games with increasing number of piles, both the definition of the games and the validity proof of their strategies seem to become more difficult. For example, whereas the union of the differences $\Delta$ between the 3 columns of the $P$-positions of Raleigh covers all of $\mathbb{Z}_{\geq 1}$, the same union for the four columns of the $P$-positions of Flora leaves out $V_{2}$. But perhaps a pattern for these games will emerge. This possibility may not be so far-fetched, since, as we saw, e.g., in $\S 4.3$, the general behavior begins only with $k=3$ (corresponding to a game with 4 piles).

Acknowledgment. Thanks to Herb Wilf, who had written to me that he defined the sequence $a^{k}(n)$ for solving part (a) of AMM problem 11238 (113 Aug-Sept 2006, 655; Sol. 115 Aug-Sept 2008, 667), and conjectured that $\Delta a^{k}(n)$ assumes only two values for every fixed $k, n \in \mathbb{Z}_{\geq 1}$. His communication and earlier work of mine - including joint work with Eric Duchêne, Richard Nowakowski and Michel Rigo, to appear in JCT-A - motivated this paper.

\section{References}

[1] J-P. Allouche and J. Shallit [2003], Automatic Sequences: Theory, Applications, Generalizations, Cambridge University Press, Cambridge.

[2] E.R. Berlekamp, J.H. Conway and R.K. Guy [2001-2004], Winning Ways for your Mathematical Plays, Vol. 1-4, A K Peters, Wellesley, MA, 2nd edition: vol. 1 (2001), vols. 2, 3 (2003), vol. 4 (2004).

[3] T.C. Brown [1993], Descriptions of the characteristic sequence of an irrational, Canad. Math. Bull. 36, 15-21.

[4] E. Duchêne and M. Rigo [2008], A morphic approach to combinatorial games: the Tribonacci case, Theor. Inform. Appl. 42, 375-393.

[5] A.S. Fraenkel [1982], How to beat your Wythoff games' opponent on three fronts, Amer. Math. Monthly 89, 353-361.

[6] A.S. Fraenkel [1985], Systems of numeration, Amer. Math. Monthly 92, $105-114$.

[7] A.S. Fraenkel [2004], Complexity, appeal and challenges of combinatorial games, Theoret. Comput. Sci. 313, 393-415. 
[8] A.S. Fraenkel [2007], The Raleigh game, in: Combinatorial Number Theory, Proc. Integers Conference 2005, in celebration of the 70th birthday of Ronald Graham, Carrollton, Georgia, Oct. 27-30, 2005; B. Landman, M. Nathanson, J. Nešetřil, R. Nowakowski, C. Pomerance, eds., de Gruyter, pp. 199-208, 2007; appeared also in Integers, Electr. J. of Combinat. Number Theory 7(2), special volume in honor of Ron Graham, \#A13, 11 pp., 2007 http://www.integers-ejcnt.org/

[9] A.S. Fraenkel and D. Krieger [2004], The structure of complementary sets of integers: a 3-shift theorem, Internat. J. Pure and Appl. Math. 10, 1-49.

[10] A.S. Fraenkel, J. Levitt and M. Shimshoni [1972], Characterization of the set of values $f(n)=[n \alpha], n=1,2, \cdots$, Discrete Math. 2, 335-345.

[11] A.S. Fraenkel, M. Mushkin and U. Tassa [1978], Determination of $\lfloor n \theta\rfloor$ by its sequence of differences, Canad. Math. Bull. 21, 441-446.

[12] A.S. Fraenkel and U. Peled, Harnessing the unwieldy MEX function, Preprint.

[13] G.H. Hardy [1999], Ramanujan: Twelve Lectures on Subjects Suggested by his Life and Work, AMS Chelsea Publishing, Providence, RI, Reprinted.

[14] G.H. Hardy and E.M. Wright [1960], An Introduction to the Theory of Numbers, 4th edition, Oxford, UK.

[15] V.E. Hoggatt and M. Bicknell-Johnson [1979], Representations of integers in terms of greatest integer functions and the golden section ratio, Fibonacci Quart. 17, 306-318.

[16] C. Kimberling [1993], Interspersions and dispersions, Proc. Amer. Math. Soc. 117, 313-321.

[17] C. Kimberling [2007], Complementary equations, J. Integer Seq. 10, 14 pp. (electronic).

[18] C. Kimberling [2008], Complementary equations and Wythoff sequences, J. Integer Seq. 11, 5 pp. (electronic).

[19] D.R. Morrison [1980], A Stolarsky array of Wythoff pairs, in: A collection of manuscripts related to the Fibonacci sequence, Fibonacci Assoc., Santa Clara, Calif., pp. 134-136.

[20] B. Obrenić, Hyperbaric numeration systems and improved Fibonacci coding, Preprint.

[21] K.A. Redish and W.F. Smyth [1991], Closed form expressions for the iterated floor function, Discrete Math. 91, 317-321.

[22] K.B. Stolarsky [1977], A set of generalized Fibonacci sequences such that each natural number belongs to exactly one, Fibonacci Quart. 15, 224. 
[23] X. Sun [2005], Wythoff's sequence and $N$-heap Wythoff's conjectures, Discrete Math. 300, 180-195.

[24] X. Sun and D. Zeilberger [2004], On Fraenkel's $N$-heap Wythoff's conjectures, Ann. Comb. 8, 225-238. 